\title{
Rolling friction of adhesive microspheres
}

\author{
S Krijt ${ }^{1}$, C Dominik ${ }^{2,3}$ and A G G M Tielens ${ }^{1}$ \\ ${ }^{1}$ Leiden Observatory, Leiden University, PO Box 9513, 2300 RA Leiden, The Netherlands \\ 2 Astronomical Institute 'Anton Pannekoek', University of Amsterdam, PO Box 94249, 1090 GE \\ Amsterdam, The Netherlands \\ ${ }^{3}$ Afdeling Sterrenkunde, Radboud Universiteit Nijmegen, Postbus 9010, 6500 GL, Nijmegen, The \\ Netherlands \\ E-mail: krijt@strw.leidenuniv.nl
}

Received 17 December 2013, revised 25 February 2014

Accepted for publication 5 March 2014

Published 10 April 2014

\begin{abstract}
The rolling friction of adhesive microspheres is an important quantity as it determines the strength and stability of larger aggregates. Current models predict rolling forces that are 1 to 2 orders of magnitude smaller than observed experimentally. Starting from the well-known Johnson-Kendall-Roberts (JKR) contact description, we derive an analytical theory for the rolling friction based on the concept of adhesion hysteresis, e.g. a difference in apparent surface energies for opening/closing cracks. We show how adhesion hysteresis causes the pressure distribution within the contact to become asymmetrical, leading to an opposing torque. Analytical expressions are derived relating the size of the hysteresis, the rolling torque, and the rolling displacement, $\xi$. We confirm the existence of a critical rolling displacement for the onset of rolling, the size of which is set by the amount of adhesion hysteresis and the size of the contact area. We demonstrate how the developed theory is able to explain the large rolling forces and particle-size dependence observed experimentally. Good agreement with experimental results is achieved for adhesion hysteresis values of $(\Delta \gamma / \gamma) \simeq 3$ for polystyrene, and $(\Delta \gamma / \gamma) \simeq 0.5$ for silicates, at crack propagation rates of $0.1 \mu \mathrm{m} \mathrm{s}^{-1}$ and $1-10 \mu \mathrm{m} \mathrm{s}^{-1}$, respectively.
\end{abstract}

Keywords: rolling friction, adhesion hysteresis, contact mechanics, viscoelasticity, microspheres

(Some figures may appear in colour only in the online journal)

\section{Introduction}

The forces between contacting micrometre-sized particles are an important ingredient in scientific studies in many different fields. Some applications include industrial issues as the transportation of powders and sands, the coagulation of aerosols in the Earth's atmosphere, and the initial steps of planet formation in proto-planetary discs around newly born stars.

As a result of the small surface-to-mass ratio of micrometre-sized bodies, attractive van der Waals forces have an important effect on the interparticle forces [1,2]. For the normal (i.e. radial) motion, the surface forces can cause the particles to adhere together and coagulate, the details of which have been studied extensively both theoretically $[3,4]$ and experimentally [5]. Lateral forces experienced during rolling or sliding have received somewhat less attention, but are equally crucial when studying the structure and strength of larger aggregates [3]. Except for very compact aggregates, the restructuring of aggregates will occur by bending arms or chains of microspheres, and individual sphere-sphere contacts act as hinge points. If little friction is associated with the rolling or sliding motion, aggregates will be weak against restructuring. More specifically, [6] have shown the compressive strength of a porous macroscopic aggregate depends directly on the rolling friction between its constituent particles.

Experimentally measuring the torque between adhesive microparticles is challenging. Still, numerous authors have succeeded using very different techniques, including manipulating single [7,8] or chains of microspheres [9] with an atomic force microscope (AFM), resolving restructuring events in time [5, 10], and non-contact techniques [11-13]. 
From the theoretical side, the rolling force between adhesive microspheres has been studied by Dominik and Tielens [14], who showed an asymmetry in the mutual contact area will give rise to an opposing torque. The size of this asymmetry was assumed to equal the interatomic distance. The theory is still widely used in $N$-body simulations studying the behaviour of large ensembles of micrometre-sized particles $[6,15,16]$, despite the fact that the parameter governing the asymmetry has to be increased by one to two orders of magnitude to match the experimental results.

In this work we set out to expand the model of [14] to allow for adhesion hysteresis and viscoelastic losses in the contact area, in an attempt to explain the large rolling forces observed experimentally. In section 2, we investigate how viscoelasticity affects the contact region, and we derive analytical expressions relating the rolling torque, the level of asymmetry of the contact, and relevant material properties. The developed theory is compared to experimental results on rolling spheres in section 3. Results are discussed in section 4 before the main conclusions are summarized in section 5 .

\section{Theory of rolling friction}

\subsection{Equilibrium adhesive contact}

To determine the rolling friction of an adhesive microsphere, a detailed knowledge is required of the contact area, and how it changes in time. Here, we briefly revisit elastic contact theory in the absence of external torques. When discussing normal forces between spheres of radii $R_{1}$ and $R_{2}$, two parameters suffice to describe the mutual contact. These are the contact radius $a$ and the distance of mutual approach $\delta$. Combining equations (5) and (11) of [17], the pressure distribution within the contact area is given as a function of $0 \leqslant r \leqslant a$ :

$$
p(r)=\frac{E^{*}}{\pi R} \frac{a^{2}-2 r^{2}+R \delta}{\sqrt{a^{2}-r^{2}}},
$$

where $R^{-1} \equiv R_{1}^{-1}+R_{2}^{-1}$ equals the effective radius, and $E^{*-1} \equiv\left(1-v_{1}^{2}\right) / E_{1}+\left(1-v_{2}^{2}\right) / E_{2}$ is the combined elastic modulus, with $E_{i}$ the Young's Moduli and $v_{i}$ the Poisson ratios of the spheres-see also [18]. We deliberately write the pressure distribution in terms of both $a$ and $\delta$, because the presence of adhesion hysteresis will lead to non-equilibrium configurations [19]. The elastic normal force between the spheres is found by integrating the pressure distribution

$$
F_{\mathrm{E}}=\int_{0}^{a} 2 \pi r p(r) \mathrm{d} r=\frac{2 E^{*}}{3 R}\left(3 a \delta R-a^{3}\right) .
$$

To describe the contact between a sphere and a flat surface, $R_{2} \rightarrow \infty$ and $R=R_{1}$ equals the sphere radius.

In the non-adhesive and perfectly elastic case, a unique relation between these two parameters exist, as described by Hertz [20]. In this limit, the elastic force is always repulsive. Almost a century later, Johnson and co-workers expanded the work of Hertz to include adhesion [1]. Their theory (hereafter JKR theory) shows that when the material's surface energy is taken into account, the contact area is enlarged compared to the Hertzian case, and contact can be maintained for negative values of $\delta$ by the formation of an adhesive neck. In addition, a stable point exists where particles stay in contact when no external force is present. At this equilibrium point, $F_{\mathrm{E}}=0$ and the contact radius is given by

$$
a_{\mathrm{eq}}=\left(\frac{9 \pi \gamma R^{2}}{2 E^{*}}\right)^{1 / 3},
$$

with $\gamma$ the combined surface energy. In JKR theory, a unique relation between $a$ and $\delta$ exists,

$$
\delta=\frac{a^{2}}{R}-\sqrt{2 \pi \gamma a / E^{*}},
$$

and in principle a single parameter suffices to describe the contact region and the interparticle force.

It should be noted that JKR theory is valid only for large values of the Tabor parameter [21]:

$$
\mu \equiv\left(\frac{R \gamma^{2}}{E^{* 2} z_{0}^{3}}\right)^{1 / 3},
$$

with $z_{0} \sim 0.2-0.4 \mathrm{~nm}$ the spacing between atoms. For $\mu>5$, JKR theory can be used, while for $\mu<0.1$ Derjaguin-MullerToporov theory is accurate [2].

In the JKR contact description, the stresses at the contact's periphery are singular. Treating the contact edge as a circular mode I crack of length $2 \pi a$, we can define a stress intensity factor as

$$
N=\lim _{r \rightarrow a} p(r) \sqrt{a-r} .
$$

The energy release associated with the creation of new surface, the 'strain energy release rate', then equals

$$
G=\pi \frac{N^{2}}{E^{*}}=\frac{E^{*}}{2 \pi} \frac{\left(a^{2}-R \delta\right)^{2}}{a R^{2}},
$$

similar to the case of rolling cylinders [22]. In the perfectly elastic case, we may identify this as the surface energy, and set $G \equiv \gamma$. When adhesion hysteresis is present however, the value of $G$ needed for crack propagation depends on whether the crack is opening $\left(G_{\mathrm{op}}>\gamma\right)$ or closing $\left(G_{\mathrm{cl}}<\gamma\right)$, and is often written as a function of opening/closing rate, i.e. the crack velocity [23-26]. The difference between the opening/closing effective surface energies $\Delta \gamma \equiv\left(G_{\mathrm{op}}-G_{\mathrm{cl}}\right)$, is called adhesion hysteresis, and can be caused in a number of ways. For viscoelastic materials, the high strain rates close to the crack tip give rise to viscoelastic hysteresis, where $(\Delta \gamma / \gamma)$ can vary between effectively zero to several orders of magnitude, depending on the rate with which the surfaces are brought together or separated [23, 27-32]. Alternatively, socalled interdigitation ${ }^{4}$ of molecular groups across the interface can give rise to substantial adhesion hysteresis, $(\Delta \gamma / \gamma)$ up to about unity, depending on the dynamics of the surface molecules involved [33-36]. For silicates, [37] have shown adhesion hysteresis can occur as a result of slow structural and chemical changes at the surface. Depending on the amount of water in the gas surrounding the silicates, the hysteresis varied significantly from 0 to $10 \mathrm{~mJ} \mathrm{~m}^{-2}$ (see figure 12 of [37]).

4 Following [35], the term interdigitation is used to describe any thermally activated processes involving molecular reorientation across an interface, including interdiffusion, interpenetration, entanglement, and any other molecular reorientation process occurring across an interface. 


\subsection{Asymmetric contact description}

When the sphere is subjected to an external torque, the sphere's centre of mass will move, and this will have an effect on the contact area itself. In the perfectly elastic case, the applied torque will cause the sphere to roll virtually without any resistance. In reality, materials are not perfectly elastic, and energy will be dissipated in the bulk of the material, and at the edges of the adhesive contact; in the remainder of the text these regions are referred to as the 'bulk' and 'crack' regions. The dissipation in both of these regions will give rise to torques opposing the rolling motion. In the remainder of this section, we focus on the torque arising from the crack region, and develop a more detailed theory of what happens to the contact during rolling. In section 4 , we give estimates for the bulk dissipation.

In rolling, the contact is asymmetric, where one side is an opening crack and the other a closing crack. Hence, it follows from (7) that the contact radius will vary for different parts of the contact region, causing it to no longer be spherical. For the contact between a cylinder and a flat, the contact region is rectangular and an analytical solution is possible [22]. In the case of a sphere, we expect $G$ to vary continuously along the periphery of the contact, as the angle between the opening/closing crack and the direction of motion changes. However, in the interest of obtaining an analytical solution, we follow [14] in approximating the contact as being comprised of two semi-circles with radii $a_{1}$ and $a_{2}$. The pressure distribution then becomes

$$
p(r)= \begin{cases}p\left(r, a_{1}, \delta\right) & \text { left semicircle, } \\ p\left(r, a_{2}, \delta\right) & \text { right semicircle, }\end{cases}
$$

where $\delta$ is the same for both halves (see also [14]), and the pressure in each half is given by (1). Note that while both halves share the same $\delta$, the radii of the two semi-circles differ, as a result of the different values of $G$ at the leading and trailing edges. It is for this reason that we avoided using an equilibrium relation between $a$ and $\delta$ in (1). There is a finite displacement between the centre of mass of the sphere (projected onto the contact surface, $x=0$ ) and the centre of the contact, given by

$$
\xi=\frac{1}{2}\left(a_{2}-a_{1}\right) \text {. }
$$

Writing $a=\left(a_{1}+a_{2}\right) / 2$, the contact can now be fully described by the three parameters $\xi, a$ and $\delta$. Figure 2(a) shows the pressure distribution of (8) for $\xi / a=0.1$, while $a$ and $\delta$ correspond to JKR equilibrium.

The contributions to the torque about the $y$-axis have been plotted in figure 2(b). From the figure it is clear that the largest torques originate close to the crack at locations furthest from the $y$-axis. The region close to $x=0$, where the assumption that the cracks are in mode I is expected to loose accuracy, has a negligible contribution. To find the (total) opposing torque that results from such a pressure distribution we have to integrate over all torque contributions in the contact area:

$$
\begin{aligned}
M & =\iint_{\text {contact }} x p(x, y) \mathrm{d} x \mathrm{~d} y \\
& =\frac{E^{*}}{4 R}\left[\left(a_{1}^{2}-\delta R\right)^{2}-\left(a_{2}^{2}-\delta R\right)^{2}\right] .
\end{aligned}
$$

With the aid of (7), the torque can be written as

$$
M=\frac{R}{2}\left(\pi a_{1} G_{1}-\pi a_{2} G_{2}\right),
$$

where $G_{1}$ and $G_{2}$ are the strain energy release rates at the trailing and leading edges of the contact. From conservation of energy, one might expect to find $M=2 R\left(a_{1} G_{1}-a_{2} G_{2}\right)$, slightly different from (11). We attribute the different prefactor to the geometry of the contact area, and the fact that different parts of the crack region contribute to the torque with different lever arms (see figure $2(b)$ ).

Realizing that, in the asymmetric case, the total elastic force equals

$$
F_{\mathrm{E}}=\frac{2 E^{*} a}{3 R}\left(3 \delta R-a^{2}-3 \xi^{2}\right),
$$

the torque of (10) can be rewritten as

$$
\begin{aligned}
M & =\left(a_{1}+a_{2}\right)\left(a_{1}-a_{2}\right) \frac{E^{*}\left(a_{1}^{2}+a_{2}^{2}-2 \delta R\right)}{4 R} \\
& =\xi\left[-F_{\mathrm{E}}+\frac{4 E^{*} a^{3}}{3 R}\right] .
\end{aligned}
$$

In the case of zero load $\left(F_{\mathrm{E}}=0\right)$ and small asymmetry, the contact size is approximately given by (3), and we obtain

$$
M=6 \pi \gamma R \xi
$$

in agreement with [14]. Lastly, it is useful to obtain a relation between the rolling displacement and the strain energy release rates. For small asymmetries, $a_{1} \simeq a_{2} \simeq a_{\mathrm{eq}}$ and we can compare (11) and (14) to find

$$
\xi=\frac{a_{\mathrm{eq}}}{12} \frac{\left(G_{1}-G_{2}\right)}{\gamma} .
$$

With this mathematical framework in place, we can now discuss what happens when an external torque is exerted on a stationary sphere.

\subsection{The onset of rolling}

The general picture is then the following. Imagine, as in figure $1(a)$, a sphere on a substrate in JKR equilibrium, so that $a_{1}=a_{2}=a_{\mathrm{eq}}$ and $G_{1}=G_{2}=\gamma$. When the sphere is subjected to an external torque, the displacement between the centre of mass and the centre of the contact will grow, as illustrated in figure $1(b)$. As a direct consequence, $a_{1}$ and $a_{2}$ will change (we will take subscript 1 to refer to the trailing half, in accordance with figure $1(b)$ ). As a result of $a_{1}$ and $a_{2}$ changing, the strain energy release rates will start to differ from $\gamma$ as dictated by (7). Initially however, $\gamma<G_{1}<G_{\text {op }}$, and the crack is unable to open at the trailing edge, effectively pinning the contact. Only when $G_{1}$ reaches $G_{\text {op }}$, crack propagation will start at the trailing edge. Setting $\left(G_{1}-G_{2}\right)=\left(G_{\mathrm{op}}-G_{\mathrm{cl}}\right) \equiv \Delta \gamma$, we can identify a critical displacement for the onset of true rolling motion:

$$
\xi_{\text {crit }}=\frac{a_{\mathrm{eq}}}{12} \frac{\Delta \gamma}{\gamma} \text {. }
$$




\section{(a): Symmetric contact}

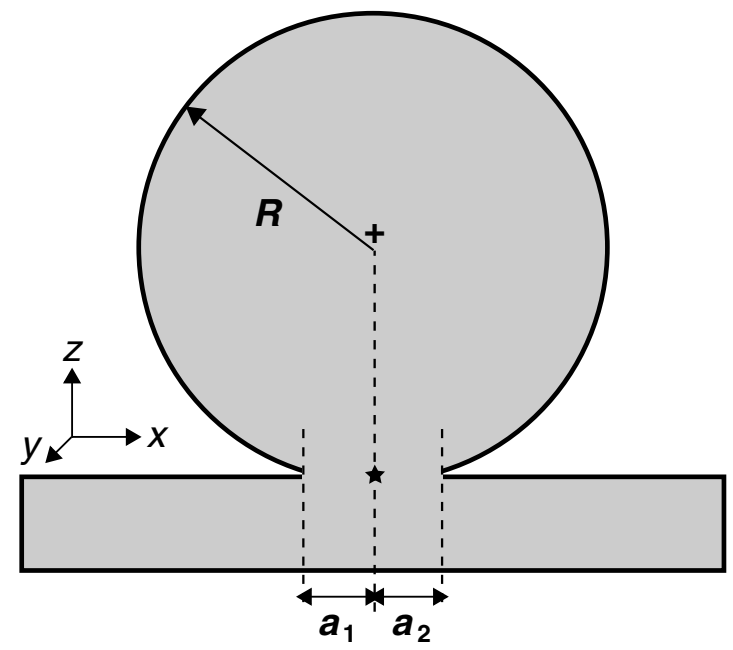

(b): Asymmetric contact

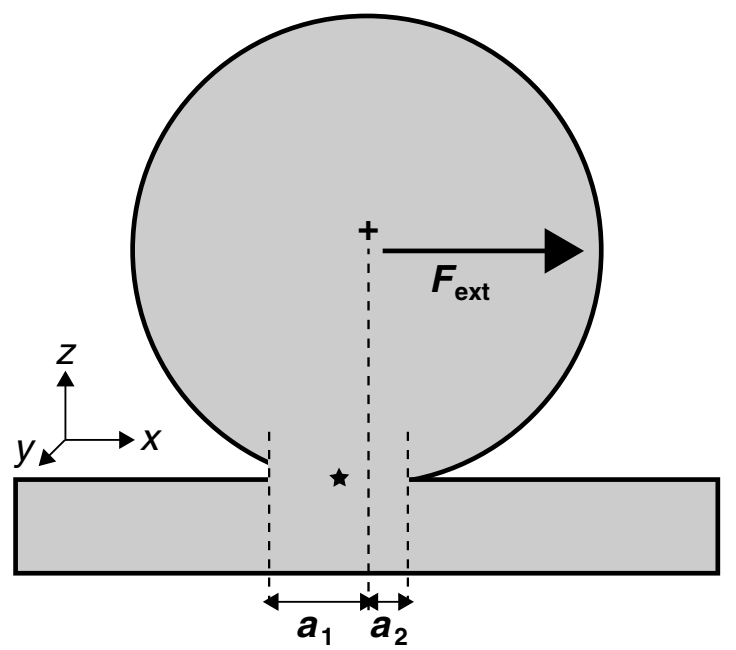

Figure 1. Side view of an adhesive sphere on a flat substrate. $(a)$ shows the symmetric case, when there are no torques acting and $a_{1}=a_{2}=a_{\mathrm{eq}}$. $(b)$ Asymmetric situation during rolling under the influence of an external force in the $x$-direction. Here, $a_{1}>a_{\mathrm{eq}}>a_{2}$. The centre of mass of the sphere $(+)$ and the centre of the contact $(\star)$ are also shown.

This relation is an important result. It reveals that the critical rolling displacement $\xi_{\text {crit }}$ is set by the size of the contact radius and the difference between the opening and closing apparent surface energy ${ }^{5}$. This picture is different from the approach of [14], where the critical displacement was assumed to equal the interatomic distance, about $0.3 \mathrm{~nm}$, independent of the particle's radius and elastic properties. If $(\Delta \gamma / \gamma)$ is constant, the critical displacement represents a fixed fraction of the contact radius, and is expected to scale with $R^{2 / 3}$. When $(\Delta \gamma / \gamma)$ varies, deviations from this slope are to be expected.

\subsection{Steady-state rolling}

When a sphere has started rolling, the opposing torque is given by (11), with the strain energy release rates now equal to $G_{\mathrm{op}}$ and $G_{\mathrm{cl}}$. Viscoelastic materials often show $G_{\mathrm{op}}$ and $G_{\mathrm{cl}}$ to depend on the crack velocity. This behaviour slightly complicates the picture described above, and it might prove necessary to solve the evolution of the sphere and the cracks in time. This method has been used by [22] for the adhesive contact of a rolling cylinder. However, in the case of a constant externally applied torque, a steady state will be realized where the contact shape is preserved and $\dot{a}_{1}=\dot{a}_{2}=0$. The external torque is then balanced by the opposing torque arising from the contact area, and the crack opening/closing velocity is equal to the velocity of the sphere's centre of mass. In this case, we can still make use of (11) developed here, realizing $\Delta \gamma$ corresponds to the adhesion hysteresis at that particular crack velocity.

It should be noted that most viscoelastic theories predict adhesion hysteresis to disappear as the crack opening/closing rate approaches zero [24, 26]. In the absence of other sources of hysteresis, this assumption would mean frictionless rolling can be achieved at infinitely low rolling velocities, and would

5 This approach was originally proposed by professor K L Johnson in a private letter to the authors of [14] in 2005. imply structures built from adhesive spheres are unstable under external forces (e.g. gravity) on very long timescales. We are not aware of such behaviour having been observed experimentally.

\subsection{Rocking motion}

Interesting behaviour is observed when the external force is removed before $\xi_{\text {crit }}$ is reached. Suppose a sphere in equilibrium receives a velocity kick in the horizontal direction at a time $t=0$. Provided the angle over which the sphere rocks is small, it can be written as $\theta=\xi / R$. The evolution of $\xi$ in time is then given by

$$
I \ddot{\xi}(t)=R M
$$

with $M$ the torque arising from the contact area, and $I=(2 / 5) m R^{2}$ the moment of inertia of the sphere. Of course, the size of the torque depends on the asymmetry of the contact. For $\xi<\xi_{\text {crit }}$, the contact is pinned and we can write

$$
\begin{aligned}
& a_{1}(t)=a_{\mathrm{eq}}-\xi(t), \\
& a_{2}(t)=a_{\mathrm{eq}}+\xi(t) .
\end{aligned}
$$

Making use of (14), the equation of motion becomes

$$
\ddot{\xi}(t)=\frac{6 \pi \gamma R^{2}}{I} \xi(t)
$$

which is readily identified as a harmonic oscillator with frequency

$$
f_{0}=\frac{1}{2 \pi}\left(\frac{6 \pi \gamma R^{2}}{I}\right)^{1 / 2}=\frac{3}{4 \pi}\left(\frac{5 \gamma}{\rho R^{3}}\right)^{1 / 2} .
$$

For microspheres, this frequency is typically of the order of $100 \mathrm{kHz}$, and has been observed experimentally [11-13]. We will discuss these experiments in section 3.1.3. 
Table 1. Material properties for silicate and PSL as used in this work. Note that for like materials, the total surface energy is twice the value listed here, which corresponds to an individual surface.

\begin{tabular}{lll}
\hline Quantity & Silicate & PSL \\
\hline$E(\mathrm{GPa})$ & 54 & 3.4 \\
$\nu(-)$ & 0.17 & 0.33 \\
$\rho\left(\mathrm{g} \mathrm{cm}^{-3}\right)$ & 2.6 & 1.026 \\
$\gamma\left(\mathrm{J} \mathrm{m}^{-2}\right)$ & 0.025 & 0.025 \\
\hline
\end{tabular}

\section{Comparison to experiments}

Now that we have a theory of rolling friction, we can compare the predictions to a number of published experiments measuring either the rolling torque or the rolling displacement. For this purpose, we will focus on two materials; polystyrene (PSL) and silicates $\left(\mathrm{SiO}_{2}\right)$, see table 1. In this section, we will discuss various experimental results within the framework of the theory developed in section 2 .

\subsection{Polystyrene microspheres}

3.1.1. Constant-velocity rolling. For PSL, [8] have measured the rolling force using an AFM for 5,10 and $15 \mu \mathrm{m}$. The spheres were pushed across a glass substrate at a constant velocity of $0.1 \mu \mathrm{m} \mathrm{s}^{-1}$. As care was taken to apply the pushing force a height $R$ from the substrate, we can relate this force to a rolling torque via $F_{\text {ext }}=M_{\text {ext }} / R$. Figure 3 shows the results of [8] in comparison to (11), for various values of $(\Delta \gamma / \gamma)$. The theory of [14], i.e. (14) with $\xi=0.3 \mathrm{~nm}$, is shown for comparison.

Figure 3 shows how the theory of section 2 explains two key features of the experimental results: the rolling force is substantially larger than expected from $\xi=0.3 \mathrm{~nm}$, and the rolling force increases with increasing radius. Indeed, a hysteresis characterized by $(\Delta \gamma / \gamma) \simeq 3$ reproduces the experimentally observed rolling force well for all three microsphere radii. For these particular particles, this corresponds to a rolling displacement of several tens of nanometres, approximately two orders of magnitude larger than $z_{0}$.

3.1.2. Critical displacement. In a somewhat similar study, Ding and co-workers moved PSL microspheres across a flat silicon substrate, by pushing the spheres with an AFM cantilever [7]. The diameters of the spheres used varied between 22.5 and $26.8 \mu \mathrm{m}$. After each push, a scanning electron microscope (SEM) image was obtained to find the new position of the microsphere, while the pushing force could be calculated from the cantilever's deflection. As a result of this experimental setup, the motion of the spheres is discontinues, and difficult to simulate in detail. An interesting results of [7] however is that a change in rolling stiffness was observed for most particles, after a total displacement between 73 and $94 \mathrm{~nm}$. Ding et al attributed this change in rolling stiffness to the transition from pre-rolling (no contact readjustment) to true rolling motion.

Figure 4 shows the observed critical rolling displacements, and compares them to predictions of (16). While the rolling displacements are orders of magnitudes larger than the atomic spacing, the results can be understood in terms of adhesion hysteresis. The inferred value of $(\Delta \gamma / \gamma) \simeq 2.5$. Unfortunately, the variation in particle radius is too small to test the correlation between $\xi_{\text {crit }}$ and $R$ predicted by (16) ${ }^{6}$.

3.1.3. Rocking microsphere. Another interesting opportunity to study the critical displacement comes in the form of the experiments conducted by Peri and Cetinkaya [11-13], where rocking motions are excited in the adhesive contact of a PSL microsphere ( $21.4 \mu \mathrm{m}$ in diameter) on various substrates. From the fact the microsphere oscillates in the lateral direction, it was inferred that the contact edges are effectively pinned (see section 2.5). Moreover, the motion is damped within a dozen oscillations, something that might well be explained by the bulk dissipative torque. The experimental results can be understood within the framework of the theory of section 2.5.

We choose to focus on the aluminum substrate, as the experimental results appear to be the cleanest (see figures 10 and 14 of [11], and use the elastic properties of table 1 of [11] and a surface energy of $0.1 \mathrm{~J} \mathrm{~m}^{-2}$. For this particular setup, $a_{\mathrm{eq}} \simeq 380 \mathrm{~nm}$. When the sphere is excited and the contact pinned, the centre of mass will oscillate with frequency $f_{0}$ set by (20). This frequency, $150 \mathrm{kHz}$ for this particular PSLaluminum system, was found by [11-13] to compare very well with the experimental observations.

The maximum observed rocking angle in the experiments ${ }^{7}$ was $0.16^{\circ}$, corresponding to $\xi=30 \mathrm{~nm}$ (two orders of magnitude larger than $z_{0}$ ), and a maximum torque of roughly $M=6 \times 10^{-13} \mathrm{Nm}$. Comparing this offset to the contact radius we find $\xi=0.08 a_{\text {eq }}$. Since this maximum $\xi<\xi_{\text {crit }}$, we can use (16) to find a lower limit of $(\Delta \gamma / \gamma)>0.95$

\subsection{Silicate microspheres}

Silicates $\left(\mathrm{SiO}_{2}\right)$ are thought to play an important role in the formation of Earth-like planets, and because of this many experiments involving silicates have been performed over the last decade or two (see $[38,39]$ for excellent reviews). Heim et al [9] measured a rolling force of $(8.5 \pm 0.3 \pm 1.3) \times 10^{-10} \mathrm{~N}$ for $1.9 \mu \mathrm{m}$-diameter silicate particles, by taking a chain of microspheres and bending it in a periodic manner. The frequency of the bending motion varied between 0.1 and $1 \mathrm{~Hz}$. Blum and Wurm [5] examined several restructuring events in microsphere structures and obtained a rolling force of $(5.0 \pm 2.5) \times 10^{10} \mathrm{~N}$, for coated $\mathrm{SiO}_{2}$ particles of $1.9 \mu \mathrm{m}$ in diameter. Lastly, using a similar approach Gundlach et al [10] measured a rolling force of $(12.1 \pm 3.6) \times 10^{-10} \mathrm{~N}$ for $1.5 \mu \mathrm{m}$ diameter $\mathrm{SiO}_{2}$.

The experimental results are compared to the adhesion hysteresis model in figure 5. At first sight, it appears the rolling force drops with particle radius. However, we attribute the scatter in rolling force values to differences in

6 In fact, the size of the particle corresponding to the left-most point in figure 4 was not determined individually, and assumed equal to the nominal sphere diameter of $21.4 \mu \mathrm{m}$ (see table 1 of [7])

7 This maximum angle was quoted for the PSL-silicon combinations, but as the values of the vertical displacement is very similar for the PSL-aluminum system (compare figures 8 and 10 of [11]) we expect it applies here as well. 

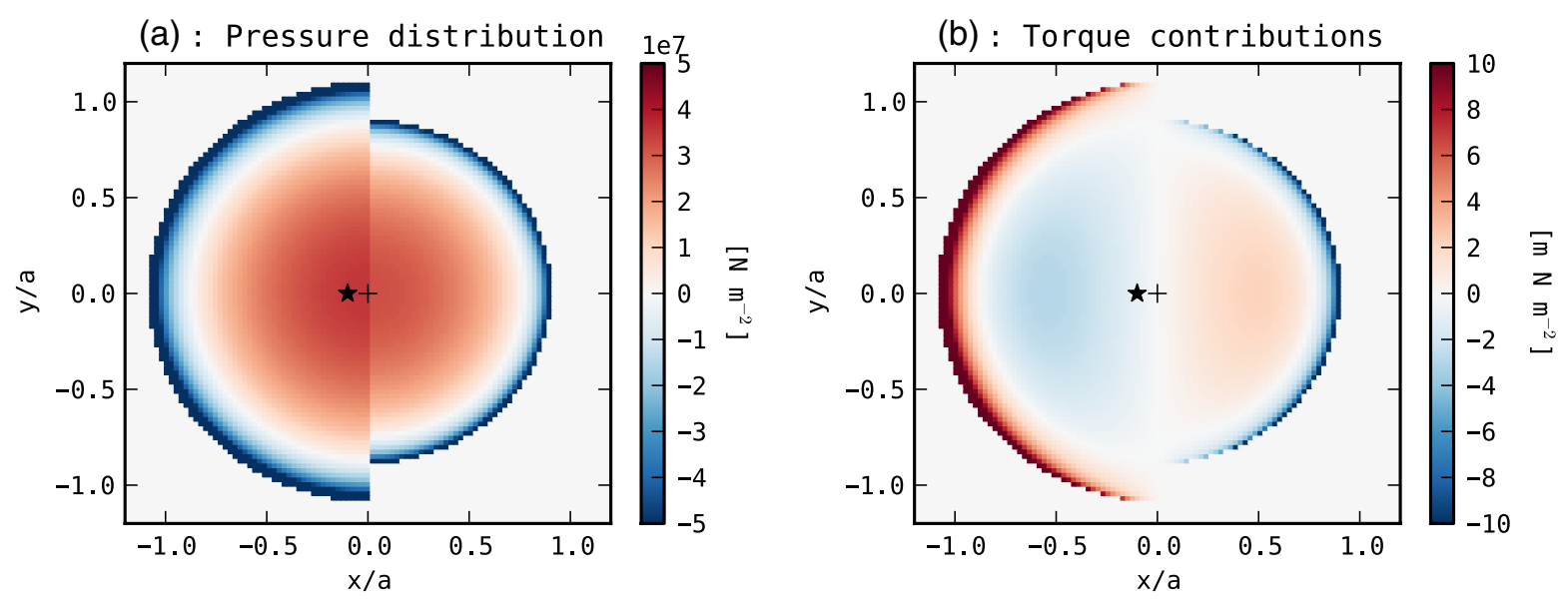

Figure 2. (a) The asymmetric pressure distribution for $\xi / a=0.1$ viewed from the top, for a sphere rolling in the $x$-direction. (b) shows the torque contributions for the same contact. The centre of mass of the sphere (+), and the centre of the contact $(\star)$ are offset. These particular numbers correspond to the contact between a PSL microsphere with radius $5 \mu \mathrm{m}$ and a PSL table.

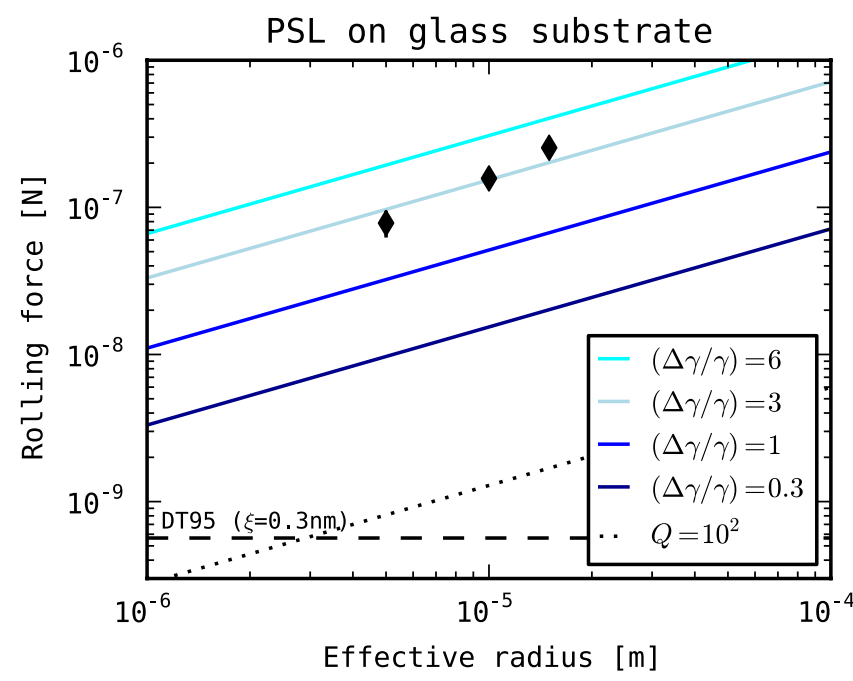

Figure 3. Experimental results of [8] ( $\checkmark)$ for the rolling force of a PSL microspheres on a glass substrate. Solid lines correspond to (11) assuming different values for $(\Delta \gamma / \gamma)$ and $a_{1}=a_{2}=a_{\mathrm{eq}}$, in which case the rolling force scales with $R^{2 / 3}$. The black dashed line corresponds to (14), assuming $\xi=0.3 \mathrm{~nm}$, and the dotted line shows the expected bulk torque for $Q=10^{2}$ (see section 4).

experimental procedures, and believe that the current data with its uncertainties is not enough to test the radius dependence of the rolling force. Nonetheless, the results can be understood in terms of $(\Delta \gamma / \gamma) \sim 0.5$. This suggests the size of the adhesion hysteresis is smaller than for the PSL of figures 3 and 4. The absolute size of the rolling displacement is significantly smaller than the one found for PSL particles in the previous section. The reason is twofold. For one, the smaller value of $(\Delta \gamma / \gamma)$ causes $\xi$ to be a smaller fraction of the contact radius. Second, the contact radius itself is much smaller for the silicate particles considered here, as they are smaller and harder.

We must address that the contact model presented here is a continuum one, and is expected to break down when the sizes of individual atoms start to play a role. In figure 5 for example, we see that for some radii, the predicted torque lies below the curve corresponding to [14]. When that occurs,

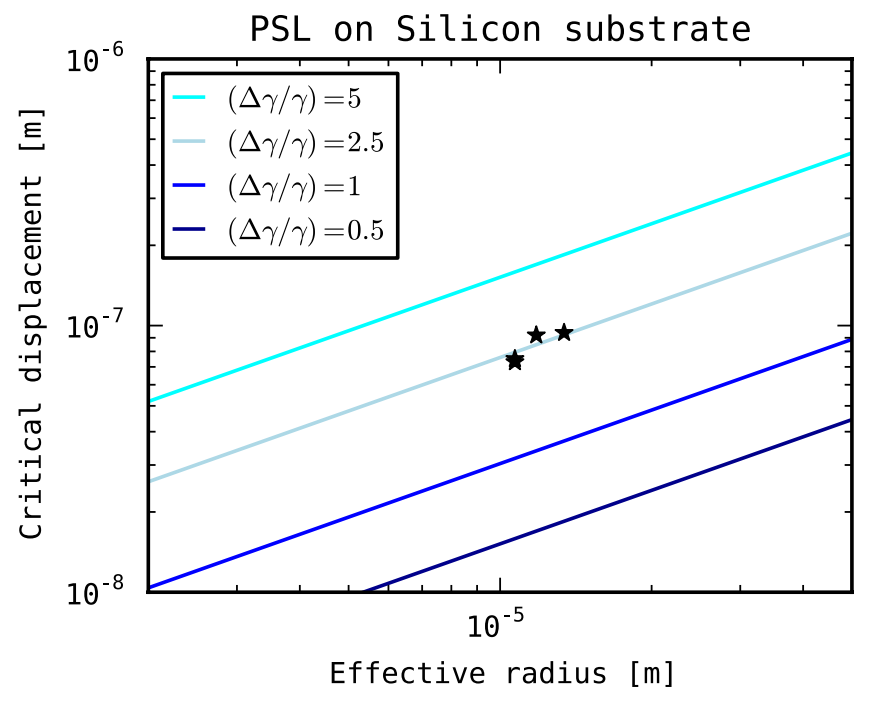

Figure 4. Experimental results of Ding et al [7] ( $\star$ ) for the critical rolling displacement of a PSL microspheres on a silicon substrate. Solid lines refer to (15), for different values of $(\Delta \gamma / \gamma)$.

the rolling displacement $\xi$ is smaller than the size of an atom, and one might question whether it is physically meaningful to have a difference between $a_{1}$ and $a_{2}$ that is arbitrarily small. The question whether continuum descriptions can still be used in this regime is beyond the scope of this work, and has to be addressed with the use of molecular dynamics simulations [40].

\section{Discussion}

In this work we propose a model for the rolling friction of microspheres based on the concept of adhesion hysteresis. In the case of rolling of a spherical contact area, the geometry does not allow for an analytical solution. However, by assuming that the contact consists of two semi-circles with different radii, an approximate analytical solution can be achieved. When the contact is close to JKR equilibrium, and the asymmetry is small compared to the contact radius itself, simple expressions relating the torque, contact asymmetry and adhesion hysteresis 


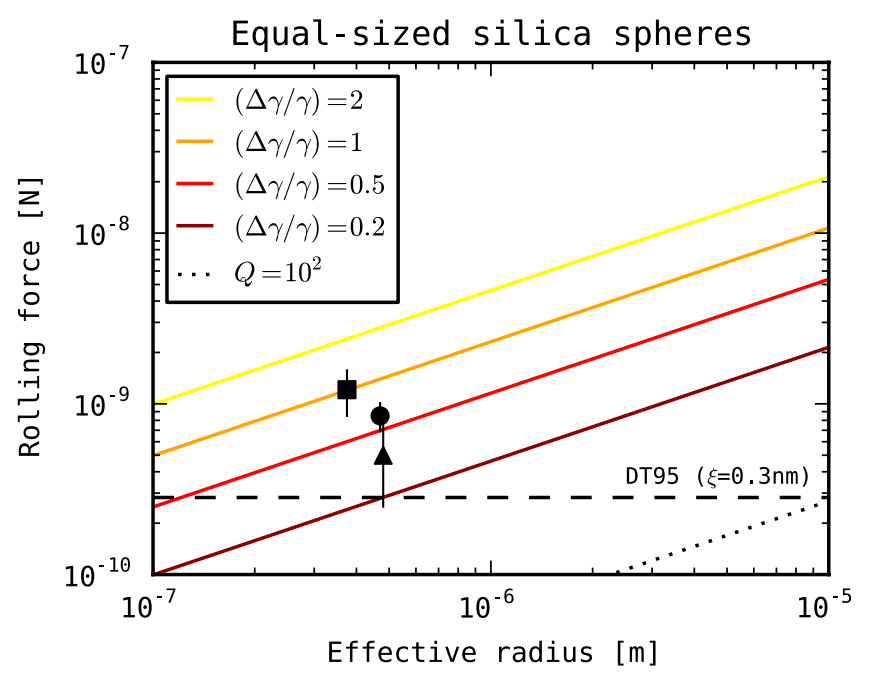

Figure 5. Rolling force versus effective radius for equal-sized silica microspheres. Symbols refer to the experimental results of [9]

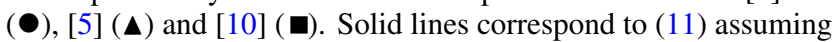
$a_{1}=a_{2}=a_{\mathrm{eq}}$ and different values for $(\Delta \gamma / \gamma)$. The black dashed line corresponds to equation (14), assuming $\xi=0.3 \mathrm{~nm}$, and the dotted line corresponds to the bulk rolling force for $Q=10^{2}$ (see section 4).

exist, and are given by (14) and (15). In the presence of external loads, the more general result (13) can be used, while the effect of the external load on the size of the contact radius and the approach should be taken into account.

Comparison of the theory to experiments of rolling microspheres reveals that the new model is capable of explaining two key results; the variation of the rolling force with particle radius, and the observation that the rolling displacement can be much larger than the interatomic distance. Good agreement between theory and experiments is achieved for adhesion hysteresis values of $(\Delta \gamma / \gamma) \simeq 3$ for PSL, and $(\Delta \gamma / \gamma) \simeq 0.5$ for silicates. When the asymmetry in the contact is small, the strain energy release rate at the contact edge is not large enough to cause the crack to move, effectively pinning the contact area. If no external forces are acting, this will cause the sphere to oscillate back and forth. An example of such behaviour is observed in the experiments of [11]. From the maximum rocking angle reached in the experiments, we $\operatorname{infer}(\Delta \gamma / \gamma)>0.95$.

Two main origins of adhesion hysteresis are mechanical, i.e. arising from viscoelastic losses near the crack tip, or chemical hysteresis, connected to the state and dynamics of surface groups and molecule chains (see section 2.1). The latter can give rise to $(\Delta \gamma / \gamma) \sim 1$, similar to the values observed in section 3. However, further comparison would require a detailed knowledge of the molecular dynamics at the surfaces involved, and is beyond the scope of this work. For viscoelastic materials, a theoretical prediction for the apparent surface energy has been obtained by [24,25]. For a threeelement solid, characterized by a single viscoelastic relaxation time, $T$, they show that the departure of $G$ from $\gamma$ is set by the non-dimensional crack velocity $v^{*} \equiv \dot{a} \gamma T /\left(z_{0}^{2} E^{*}\right)$, with $\dot{a}$ the crack opening/closing velocity. A difference in apparent surface energy of 3 and 0.5 then corresponds to a $v^{*}$ of 2 and 0.1, respectively (through figure 6 of [24]).
For the experiments of [8], the crack velocity equals the rolling velocity of $10^{-7} \mathrm{~m} \mathrm{~s}^{-1}$. For the experiments plotted in figure 5 , the rolling velocities are estimated ${ }^{8}$ to lie between $10^{-6}-10^{-5} \mathrm{~m} \mathrm{~s}^{-1}$. Plugging in the material properties and correct $\dot{a}$ returns $T=7 \times 10^{-2}$ s for the PSL particles of figure 3, and $T=5 \times 10^{-4} \mathrm{~s}$ for the silicates of figure 5 . For PSL at room temperature, the obtained $T^{-1}$ does not coincide with either $\alpha$ or $\beta$ relaxation peaks, which occur at much lower frequencies (figure 8.20 of [41]), but might correspond to one of several other relaxation peaks [42]. Alternatively, much shorter relaxation timescales were obtained by [19] by fitting rebound experiments of micrometre-sized spheres, indicating that a viscoelastic model with a single characteristic relaxation time might not accurately describe the material response over a large range of strain frequencies. However, as there is virtually no variation in rolling velocity in the experiments used in section 3 , connecting the observed adhesion hysteresis to a viscous relaxation time remains speculative.

Thus, measurements of the rolling friction of adhesive spheres provide a powerful window into adhesion hysteresis of the materials involved, as the observed torque can be directly related to the difference in apparent surface energies on both sides of the contact. So-called JKR experiments (e.g. $[36,43])$ are also powerful, but require knowledge of the contact size throughout the loading-unloading experiment, which is challenging for the relatively small contacts between microspheres. Additional experiments, that measure the rolling force of microspheres in a controlled manner, will provide valuable insight in the behaviour at the crack tip. Specifically, experiments probing a broad range of rolling velocities will allow a more thorough comparison to viscoelastic crack theory.

So far, we have neglected the effects of bulk energy dissipation on the rolling torques. The elastic energy stored in a spherically symmetric contact equals $[17,44]$

$$
U_{\mathrm{E}}=\frac{E^{*} a^{3}}{3 R}\left[\delta\left(\frac{3 \delta R}{a^{2}}-1\right)-\frac{a^{2}}{5 R}\left(\frac{5 \delta R}{a^{2}}-3\right)\right],
$$

where again we have deliberately omitted making use of an equilibrium relation between $a$ and $\delta$. Assume now the sphere rolls over a distance $2 a$. In that case, a surface element that passed through the contact area has undergone a complete stress cycle. Assuming the bulk dissipation is small, the total energy loss associated with the elastic strain energy can then be estimated as $U_{\mathrm{B}} \sim(\pi / Q) U_{\mathrm{E}}$, with $Q$ the 'quality factor' ${ }^{\prime}$. The reciprocal of the quality factor is associated with the ratio of energy dissipated to the energy stored in dynamic loading, and can be viewed as a fundamental measure of mechanical dissipation [45]. In the case of rolling friction, the reciprocal of the quality factor is identical to Tabor's hysteresis loss factor [46], apart from a numerical factor of order unity [18]. Typical values for the quality factor in solids are $10-10^{2}$ for polymers, $10^{3}$ for glass and soft metals, but may vary with

8 These numbers are obtained by estimating the rolling angle and timescales from figures 3 of [5] and 7 of [10].

9 In treatises of viscoelastic materials, the loss tangent $\tan \delta$ is often used. To avoid confusion with the mutual approach, we will use the quality factor instead. These are related through $Q^{-1} \simeq \tan \delta$. 
frequency. As the sphere rolled over $2 a$, the torque associated with this bulk energy loss can be written as ${ }^{10}$

$$
M_{\mathrm{B}}=\frac{U_{\mathrm{B}} R}{2 a} \sim \frac{\pi U_{\mathrm{E}}}{Q} \frac{R}{2 a} .
$$

Earlier we obtained the torque arising from the region close to the crack. Making use of (11) while plugging in $a_{1} \sim a_{2} \sim a_{\mathrm{eq}}$ and the surface energy $U_{\mathrm{S}}=-\pi a_{\mathrm{eq}}^{2} \gamma$, we can compare the sizes of the two torques to find

$$
\frac{M_{\mathrm{B}}}{M_{\mathrm{C}}} \sim\left(\frac{U_{\mathrm{E}}}{U_{\mathrm{S}}}\right)\left(\frac{\pi}{Q}\right)\left(\frac{\gamma}{\Delta \gamma}\right) .
$$

While approximate, the above expression is very instructive; and we will briefly discuss the three terms on the right-hand side of (23). Firstly, in a JKR equilibrium contact, the total energy is always negative, as the particles are bound. This immediately tells us the surface energy dominated the elastic energy. If external forces are present, the elastic energy can be increased significantly, potentially changing the value of $\left(U_{\mathrm{E}} / U_{\mathrm{S}}\right)$. In the specific case of a sphere resting on a flat surface, the gravitational force on the sphere is such an external force. For a silicate sphere, gravity will cause significant deviation from the JKR equilibrium only for sphere radii larger than a millimetre. Alternatively, the potential presence of dust or asperities can decrease the effective contact area, and reduce the overall importance of the surface energy and adhesion [21]. Secondly, the ratio $(\pi / Q)$ is very small for solids. At different frequencies, different mechanisms will be responsible for demoing, and the behaviour of $Q$ as a function of frequency will differ per material. A wide range of experimental techniques are used across the frequency spectrum to measure $Q$ (figure 6.25 of [45]), but typical values at $\mathrm{Hz}$ to $\mathrm{kHz}$ frequencies and at room temperatures are $10-10^{2}$ for polymers, $10^{3}$ for glass and soft metals, and orders of magnitude larger for structural metals and quartz ( [45, p 208]). In a typical crystalline solid, the quality factor is relatively constant as a function of frequency, while the $Q$-spectrum of a typical amorphous polymer shows more pronounced peaks, corresponding to various molecular motions (figures 8.1 and 8.2 of [45]). Thus, we expect $\left(M_{\mathrm{B}} / M_{\mathrm{C}}\right) \ll 1$ for small and smooth adhesive spheres in a JKR equilibrium contact, justifying the approach of section 2 . This finding is supported by experiments on adhesive cylinders $[22,49,50]$, where the observed rolling torque was attributed solely to adhesion hysteresis. Alternatively, the presence of asperities, additional external loads, very low quality factors, or negligible adhesion hysteresis, will act to increase the relative importance of the bulk dissipation.

From the damped oscillation in the experiment of Peri and Cetinkaya (see section 3.1.3), we can derive an order-ofmagnitude number for the quality factor. First, we modify the

\footnotetext{
${ }^{10}$ In the Hertzian case, (22) is in agreement with the result of Brilliantov and Pöschel $[47,48]$, who obtained an expression for the rolling torque for non-adhesive elastic spheres by integrating small dissipative stresses in the contact area, provided their viscoelastic constant, $A$, is related to the quality factor through $Q \sim\left(2 a / v_{\text {roll }}\right) / A$. This relation is expected since $A$ has units of time, and $\left(2 a / v_{\text {roll }}\right)$ is the typical stress timescale probed by the moving contact.
}

expression for the total elastic energy to include the contact asymmetry

$$
U_{\mathrm{E}}\left(a_{1}, a_{2}, \delta\right)=\frac{1}{2}\left[U_{\mathrm{E}}\left(a_{1}, \delta\right)+U_{\mathrm{E}}\left(a_{2}, \delta\right)\right],
$$

where $U_{\mathrm{E}}\left(a_{i}, \delta\right)$ is given by $(21)$. Using this relation, we see that for an asymmetry of $\xi / a=0.08$, the elastic energy is increased by about $\Delta U_{\mathrm{E}}=0.1 U_{\mathrm{E}}$ compared to the symmetric case. During the oscillation, this energy is converted into rocking motion and vice versa, and in a single oscillation to a maximum $\xi$ and back, a fraction $\pi / Q$ of $\Delta U_{\mathrm{E}}$ is dissipated. Now, we can write down a condition for the timescale on which this excess energy is dissipated, and the oscillation is stopped. It is instructive to express this timescale in a number of periods:

$$
f_{0} \frac{\Delta U_{\mathrm{E}}}{(\mathrm{d} / \mathrm{d} t) \Delta U_{\mathrm{E}}} \sim \frac{Q}{\pi} .
$$

From the observation that the oscillations is damped in roughly 10 periods, we obtain $Q \sim 30$, in agreement with typical quality factors of polymeric materials at $\mathrm{kHz}$ frequencies [45], strengthening our assumption that the damping originates from energy dissipation in the bulk of the material.

\section{Conclusions}

Our main findings can be summarized as follows:

- For microspheres in JKR equilibrium, rolling friction will be dominated by dissipation associated with the opening and closing of the cracks on both sides of the contact region (e.g. adhesion hysteresis).

- For a fixed adhesion hysteresis $(\Delta \gamma / \gamma)$, the critical rolling displacement equals a constant fraction of the contact radius, and therefore scales with $R^{2 / 3}$.

- The theory is capable of reproducing a variety of experimental results, explaining in a natural way the large observed values of the rolling force and rolling displacement during pushing experiments (figures 3, 4 and 5); the rocking motion of microspheres for small rolling angles, and the observed radius dependence of the rolling force (figure 3 ).

- Applying the theory to experimental results indicates adhesion hysteresis for polystyrene roughly equals $(\Delta \gamma / \gamma) \simeq 3$, and $(\Delta \gamma / \gamma) \simeq 0.5$ for silicate particles.

Owing to their simple forms, (14) and (15) can be directly integrated in simulations of systems of adhesive spheres, while future experiments measuring the rolling forces for different velocities and particle sizes can be used to test and discriminate between different models of adhesion hysteresis.

\section{Acknowledgments}

Dust studies at Leiden Observatory are supported through the Spinoza Premie of the Dutch Science Agency, NWO. The authors would like to thank professor K L Johnson for pointing out the relation between rolling friction and adhesion hysteresis in an adhesive contact. The authors want to thank the anonymous referees, whose comments have helped to improve the manuscript. 


\section{References}

[1] Johnson K L, Kendall K L and Roberts A D 1971 Proc. R. Soc. A 324 301-13

[2] Derjaguin B V, Muller V M and Toporov Y P 1975 J. Colloid Interface Sci. 53 314-26

[3] Dominik C and Tielens A G G M 1997 Astrophys. J. 480647

[4] Kempf S, Pfalzner S and Henning T K 1999 Icarus 141 388-98

[5] Blum J and Wurm G 2000 Icarus 143138

[6] Kataoka A, Tanaka H, Okuzumi S and Wada K 2013 Astron. Astrophys. 554 A4

[7] Ding W, Howard A J, Peri M D M and Cetinkaya C 2007 Phil. Mag. 87 5685-96

[8] Sumer B and Sitti M 2008 J. Adhes. Sci. Technol. 22481

[9] Heim L-O, Blum J, Preuss M and Butt H-J 1999 Phys. Rev. Lett. 833328

[10] Gundlach B, Kilias S, Beitz E and Blum J 2011 Icarus 214717

[11] Peri M D M and Cetinkaya C 2005 J. Colloid Interface Sci. 288432

[12] Peri M D M and Cetinkaya C 2005 Phil. Mag. 851347

[13] Peri M D M and Cetinkaya C 2005 Appl. Phys. Lett. 86194103

[14] Dominik C and Tielens A G G M 1995 Phil. Mag. A 72783

[15] Okuzumi S, Tanaka H, Kobayashi H and Wada K 2012 Astrophys J. $\mathbf{7 5 2} 106$

[16] Seizinger A, Krijt S and Kley W 2013 Astron. Astrophys. 560 A 45

[17] Muller V M, Yushchenko V S and Derjaguin B V 1980 J. Colloid Interface Sci. 77 91-101

[18] Johnson K L 1985 Contact Mechanics (Cambridge: Cambridge University Press)

[19] Krijt S, Güttler C, Heißelmann D, Dominik C and Tielens A G G M 2013 J. Phys. D: Appl. Phys. 46435303

[20] Hertz H 1882 J. Reine Angew. Math. 92 156-71

[21] Tabor D 1977 J. Colloid Interface Sci. 58 2-13

[22] Greenwood J A, Johnson K L, Choi S-H and Chaudhury M K 2009 J. Phys. D: Appl. Phys. 42035301

[23] Maugis D and Barquins M 1978 J. Phys. D: Appl. Phys. 111989

[24] Greenwood J A 2004 J. Phys. D: Appl. Phys. 372557

[25] Greenwood J A and Johnson K L 2006 J. Colloid Interface Sci. 296 284-91
[26] Barthel E and Frétigny C 2009 J. Phys. D: Appl. Phys. 42195302

[27] Schapery R A 1975 Int. J. Fracture 11 141-59

[28] Schapery R A 1975 Int. J. Fracture 11 369-88

[29] Greenwood J A and Johnson K L 1981 Phil. Mag. A 43 697-711

[30] Schapery R A 1989 Int. J. Fracture 39 163-89

[31] Hui C-Y, Baney J M and Kramer E J 1998 Langmuir 14 6570-8

[32] Baney J M and Hui C-Y 1999 J. Appl. Phys. $864232-41$

[33] Chen Y-L, Helm C A and Israelachvili J N 1991 J. Phys. Chem. 95 10736-47

[34] Chaudhury M K and Whitesides G M 1991 Langmuir 7 1013-25

[35] Yoshizawa H, Chen Y-L and Israelachvili J 1993 J. Phys. Chem. 97 4128-40

[36] Chaudhury M K and Owen M J 1993 J. Phys. Chem. $975722-6$

[37] Vigil G, Xu Z, Steinberg S and Israelachvili J 1994 J. Colloid Interface Sci. 165 367-85

[38] Blum J and Wurm G 2008 Annu. Rev. Astron. Astrophys. 4621

[39] Güttler C, Blum J, Zsom A, Ormel C W and Dullemond C P 2010 Astron. Astrophys. 513 A56

[40] Tanaka H, Wada K, Suyama T and Okuzumi S 2012 Prog. Theor. Phys. 195101

[41] Sperling L H 2007 Introduction to Physical Polymer Science 4th edn (New York: Wiley)

[42] Yano O and Wada Y 1971 J. Polym. Sci. A $9669-86$

[43] Lorenz B, Krick B A, Mulakaluri N, Smolyakova M, Dieluweit S, Sawyer W G and Persson B N J 2013 J. Phys.: Condens. Matter 25225004

[44] Billiantov N V, Albers N, Spahn F and Pöschel T 2007 Phys. Rev. E 76051302

[45] Lakes R 2009 Viscoelastic Materials (Cambridge: Cambridge University Press)

[46] Tabor D 1955 Proc. R. Soc. A 229 198-220

[47] Brilliantov N V and Pöschel T 1998 Europhys. Lett. 42511

[48] Brilliantov N V and Pöschel T 1999 Eur. Phys. J. B 12299

[49] Maugis D 1985 J. Mater. Sci. $203041-73$

[50] She H, Malotky D and Chaudhury M K 1998 Langmuir 14 3090-100 